\title{
STOCHASTIC ORDERINGS AND AGEING PROPERTIES OF RESIDUAL LIFE LENGTHS OF LIVE COMPONENTS IN $(n-k+1)$-OUT-OF- $n$ SYSTEMS
}

\author{
NARAYANASWAMY BALAKRISHNAN, ${ }^{*}$ McMaster University \\ GHOBAD BARMALZAN ${ }^{* *}$ AND \\ ABEDIN HAIDARI, ${ }^{* *}$ Zabol University
}

\begin{abstract}
Suppose that a system consists of $n$ independent and identically distributed components and that the life lengths of the $n$ components are $X_{i}, i=1, \ldots, n$. For $k \in\{1, \ldots, n-1\}$, let $X_{1}^{(k)}, \ldots, X_{n-k}^{(k)}$ be the residual life lengths of the live components following the $k$ th failure in the system. In this paper we extend various stochastic ordering results presented in Bairamov and Arnold (2008) on the residual life lengths of the live components in an $(n-k+1)$-out-of- $n$ system, and also present a new result concerning the multivariate stochastic ordering of live components in the two-sample situation. Finally, we also characterize exponential distributions under a weaker condition than those introduced in Bairamov and Arnold (2008) and show that some special ageing properties of the original residual life lengths get preserved by residual life lengths.
\end{abstract}

Keywords: $(n-k+1)$-out-of- $n$ system; stochastic ordering; multivariate mixture; decreasing hazard rate; live component; order statistics

2010 Mathematics Subject Classification: Primary 60E15

Secondary 90B25

\section{Introduction}

Consider a system with $n$ components, and suppose that this system fails if and only if at least $k$ components fail. Such a system is said to be an $(n-k+1)$-out-of- $n$ system. Evidently, the lifetime of an $(n-k+1)$-out-of- $n$ system is equal in distribution to the $k$ th order statistic from a sample of size $n$. So, by using the theory of order statistics, many properties of $(n-k+1)$ out-of- $n$ systems have been established in the literature. Specifically, some well-known ageing properties and stochastic ordering results have been developed.

The stochastic comparison of order statistics was initiated in Pledger and Proschan (1971) and since then the theory has attracted the attention of numerous researchers. Interested readers are referred to Proschan and Sethuraman (1976), Boland et al. (1994), Boland et al. (1998), Khaledi and Kochar (2000b), Paltanea (2008), Zhao et al. (2009), and Zhao and Balakrishnan (2009). In an $(n-k+1)$-out-of- $n$ system, just after the $k$ th failure has been observed, there will be $n-k$ components still alive in the system. Thus, we have two sets of random variables, the first consisting of the original lifetimes and the second consisting of the residual life lengths of the live components. In this regard, Bairamov and Arnold (2008) studied distributional properties of the residual life lengths of live units and obtained their joint distribution. They also characterized exponential distributions under some conditions and showed that, under some

Received 28 May 2012; revision received 23 February 2013.

* Postal address: Department of Mathematics and Statistics, McMaster University, Hamilton, Ontario, L8S 4K1,

Canada. Email address: bala@mcmaster.ca

** Postal address: Department of Statistics, Zabol University, Sistan and Baluchestan, Iran. 
well-known ageing properties of the original component lifetime distribution, one can compare residual life lengths of the live components with the original lifetimes in terms of the usual stochastic ordering. We extend their results here, and also establish a multivariate stochastic ordering result.

The rest of this paper proceeds as follows. In Section 2 we review the concepts of some specific stochastic orderings and ageing properties. In Section 3 we present some extensions of the results of Bairamov and Arnold (2008) from the usual stochastic ordering to the mean residual life order, hazard rate order, dispersive order, and likelihood ratio order. In Section 4 we characterize exponential distributions under a weaker condition than those introduced in Bairamov and Arnold (2008), and show that if the residual life lengths are uncorrelated, then the original component lifetime distribution is exponential. In Section 5 we study some ageing properties of the residual life lengths. Finally, in Section 6 we present a new result about the multivariate stochastic ordering of the residual lifetimes of live components in the two-sample situation. Throughout this paper, we assume that the failure of one component does not affect the functioning of the remaining components in the system. If this assumption is not true then models involving the so-called sequential order statistics (see Kamps (1995) and Cramer and Kamps (1996)) need to be considered. We do not consider this set-up here, and we shall reserve it for a future study. We assume that all of the distributions under study are absolutely continuous with common support $(0, \infty)$, and also adopt the phrasing increasing for nondecreasing and decreasing for nonincreasing.

\section{Definitions and notation}

In this section we recall the definitions of some well-known ageing properties and stochastic orderings that are pertinent to the developments in this paper.

Definition 1. Suppose that the distribution function $F$ is absolutely continuous with density function $f$ such that $f(x)=0$ for $x<0$.

(i) We say that $F$ is new better than used (NBU) if

$$
\bar{F}(x+t) \leq \bar{F}(x) \bar{F}(t) \text { for all } t, x \geq 0,
$$

where $\bar{F}:=1-F$. If inequality (1) is reversed then $F$ is said to be new worse than used (NWU).

(ii) We say that the distribution $F$ (with finite mean) has a decreasing mean residual life ( $F$ is DMRL) if

$$
m_{F}(t)=\frac{1}{\bar{F}(t)} \int_{t}^{\infty} \bar{F}(x) \mathrm{d} x \quad \text { is decreasing in } t \geq 0 .
$$

Similarly, $F$ has an increasing mean residual life ( $F$ is IMRL) if $m_{F}(t)$ is increasing in $t \geq 0$.

(iii) We say that the distribution $F$ has an increasing (decreasing) hazard rate, written $F$ is IHR (DHR), if $\bar{F}(x+t) / \bar{F}(t)$ is decreasing (increasing) as a function of $t$ for all $x \geq 0$. Equivalently, $F$ is IHR (DHR) if its hazard rate function is increasing (decreasing).

(iv) We say that $F$ has a log-concave (log-convex) density, written $f$ is log-concave (logconvex), if $f(x+t) / f(t)$ is decreasing (increasing) as a function of $t$ for all $x \geq 0$. In other words, $f$ is $\log$-concave (log-convex) if $\log f$ is concave (convex). 
The following implications between all these ageing properties are well known:

$$
\begin{aligned}
& \text { log-concave density } \Longrightarrow \mathrm{IHR} \Longrightarrow \mathrm{DMRL} \Longrightarrow \mathrm{NBU} \text {, } \\
& \text { log-convex density } \Longrightarrow \text { DHR } \Longrightarrow \text { IMRL } \Longrightarrow \text { NWU. }
\end{aligned}
$$

For comprehensive discussions on ageing properties and relations between them, we refer the reader to Barlow and Proschan (1981), Marshall and Olkin (2007), and Lai and Xie (2006). Now, we briefly describe some notion for univariate and multivariate stochastic orderings which is most pertinent to the results established in the subsequent sections.

Definition 2. For two random variables $X$ and $Y$ with densities $f$ and $g$, distribution functions $F$ and $G$, and right-continuous inverses (quantile functions) $F^{-1}$ and $G^{-1}$, respectively, let $\bar{F}=1-F$ and $\bar{G}=1-G$ denote the corresponding survival functions. With the ratios in the statements below being well defined.

(i) $X$ is said to be smaller than $Y$ in the likelihood ratio order (denoted by $X \leq \operatorname{lr} Y$ ) if $g(x) / f(x)$ is increasing in $x$.

(ii) $X$ is said to be smaller than $Y$ in the hazard rate order (denoted by $X \leq_{\mathrm{hr}} Y$ ) if $\bar{G}(x) / \bar{F}(x)$ is increasing in $x$. If $r_{X}\left(r_{Y}\right)$ denotes the hazard rate function of $X(Y)$, then $X \leq_{\mathrm{hr}} Y$ if and only if $r_{Y}(x) \leq r_{X}(x)$.

(iii) $X$ is said to be smaller than $Y$ in the usual stochastic order (denoted by $X \leq_{\text {st }} Y$ ) if $\bar{F}(x) \leq \bar{G}(x)$. For all increasing functions $\phi: \mathbb{R} \rightarrow \mathbb{R}, X \leq_{\text {st }} Y$ if and only if $\mathbb{E}(\phi(X)) \leq \mathbb{E}(\phi(Y))$ when the expectations exist.

(iv) $X$ is said to be smaller than $Y$ in the dispersive order (denoted by $X \leq_{\operatorname{disp}} Y$ ) if

$$
F^{-1}(\beta)-F^{-1}(\alpha) \leq G^{-1}(\beta)-G^{-1}(\alpha) \text { for } 0 \leq \alpha<\beta \leq 1 .
$$

(v) $X$ is said to be smaller than $Y$ in the mean residual life order (denoted by $X \leq{ }_{\mathrm{mrl}} Y$ ) if, for all $t \geq 0$,

$$
\frac{\int_{t}^{\infty} \bar{F}(u) \mathrm{d} u}{\bar{F}(t)} \leq \frac{\int_{t}^{\infty} \bar{G}(u) \mathrm{d} u}{\bar{G}(t)} .
$$

The following implications between these orderings are well known:

$$
X \leq_{\mathrm{lr}} Y \quad \Longrightarrow \quad X \leq_{\mathrm{hr}} Y \quad \Longrightarrow \quad X \leq_{\mathrm{mrl}} Y \text { and } X \leq_{\mathrm{st}} Y .
$$

Note that if $X \leq_{\text {disp }} Y$ and $X$ and $Y$ have finite equal left-end support points, then $X \leq_{\text {st }} Y$. When $X$ and $Y$ have finite equal right-end support points, then $X \leq_{\operatorname{disp}} Y$ implies that $Y \leq_{\mathrm{st}} X$.

Definition 3. We say that the random vector $\boldsymbol{X}=\left(X_{1}, \ldots, X_{n}\right)$ is smaller than the random vector $\boldsymbol{Y}=\left(Y_{1}, \ldots, Y_{n}\right)$ in the multivariate stochastic order (denoted by $\left.\boldsymbol{X} \leq_{\text {st }} \boldsymbol{Y}\right)$ if

$$
\mathbb{E}(\phi(\boldsymbol{X})) \leq \mathbb{E}(\phi(\boldsymbol{Y})),
$$

for all increasing functions $\phi: \mathbb{R}^{n} \rightarrow \mathbb{R}$ for which the expectations exist.

It is easy to see that multivariate stochastic ordering implies componentwise usual stochastic ordering. Interested readers are referred to Müller and Stoyan (2002) and Shaked and Shanthikumar (2007) for comprehensive discussions on univariate and multivariate stochastic orderings. 


\section{Residual life lengths of live components in the one-sample situation}

Let us consider an $(n-k+1)$-out-of- $n$ system in which the lifetimes $X_{1}, \ldots, X_{n}$ of the $n$ components in the system are i.i.d. with common absolutely continuous distribution function $F$ and corresponding density function $f$. When the $k$ th failure has been observed, at which time the $(n-k+1)$-out-of- $n$ system fails, there are $n-k$ components still alive. Suppose that $X_{1}^{(k)}, \ldots, X_{n-k}^{(k)}$ denote the residual life lengths of these $n-k$ live components when the system fails. Bairamov and Arnold (2008) showed that the joint survival function of the residual life lengths is given by

$$
\bar{H}^{(k)}\left(x_{1}, \ldots, x_{n-k}\right)=\mathbb{P}\left(X_{1}^{(k)}>x_{1}, \ldots, X_{n-k}^{(k)}>x_{n-k}\right)=\int_{0}^{\infty} \prod_{j=1}^{n-k} \frac{\bar{F}\left(x_{j}+t\right)}{\bar{F}(t)} f_{k: n}(t) \mathrm{d} t,
$$

where $f_{k: n}(t)$ is the probability density function of the $k$ th order statistic $X_{k: n}$; see Arnold et al. (1992) and David and Nagaraja (2003) for details on distributional properties and applications of order statistics. From (2), it is easy to see that the residual life lengths $X_{1}^{(k)}, \ldots, X_{n-k}^{(k)}$ are exchangeable and, consequently, they have the same marginal and joint distributions. The common marginal survival function of $X_{i}^{(k)}$ is readily seen from (3) to be

$$
\bar{H}(x)=\int_{0}^{\infty} \frac{\bar{F}(x+t)}{\bar{F}(t)} f_{k: n}(t) \mathrm{d} t,
$$

and the corresponding marginal density function of $X_{i}^{(k)}$ is

$$
h(x)=\int_{0}^{\infty} \frac{f(x+t)}{\bar{F}(t)} f_{k: n}(t) \mathrm{d} t .
$$

Note that, in general, the residual life lengths of the live components are not independent, but conditionally independent given the time of the $k$ th failure. Suppose that, for $i=1, \ldots, n-k$, $X_{i: n-k}^{(k)}$ denotes the $i$ th order statistic of the residual life lengths. Then, we have

$$
X_{i: n-k}^{(k)}=X_{i+k: n}-X_{k: n}, \quad i=1, \ldots, n-k .
$$

It is reasonable to expect that when components get better with the passing of time, the residual life lengths of live components become stochastically larger than the original lifetimes. Bairamov and Arnold (2008) have in fact proved this property in the form of the following proposition.

Proposition 1. If $F$ is $N W U(N B U)$ then $X_{1} \leq_{\mathrm{st}}\left(\geq_{\mathrm{st}}\right) X_{1}^{(k)}$.

Continuing in the same way, under some well-known ageing properties of the original lifetime distribution, we prove in the following theorem some other stochastic orderings between original lifetimes and residual life lengths of live components.

Theorem 1. We have the following ordering results.

(i) If $F$ is IMRL (DMRL) then $X_{1} \leq_{\mathrm{mrl}}\left(\geq_{\mathrm{mrl}}\right) X_{1}^{(k)}$.

(ii) If $F$ is DHR (IHR) then $X_{1} \leq_{\mathrm{hr}}\left(\geq_{\mathrm{hr}}\right) X_{1}^{(k)}$.

(iii) If $F$ is DHR then $X_{1} \leq \operatorname{disp} X_{1}^{(k)}$.

(iv) If $f$ is log-convex (log-concave) then $X_{1} \leq_{\mathrm{lr}}\left(\geq_{\mathrm{lr}}\right) X_{1}^{(k)}$. 
Proof. (i) Let $m_{H}(u)$ and $m_{F}(u)$ denote the mean residual life functions of $X_{1}^{(k)}$ and $X_{1}$, respectively. The mean residual life function of $X_{1}^{(k)}$ can be derived as

$$
\begin{aligned}
m_{H}(u) & =\int_{u}^{\infty} \frac{\bar{H}(x)}{\bar{H}(u)} \mathrm{d} x \\
& =\int_{u}^{\infty} \int_{0}^{\infty} \frac{\bar{F}(x+t)}{\bar{H}(u) \bar{F}(t)} f_{k: n}(t) \mathrm{d} t \mathrm{~d} x \\
& =\int_{0}^{\infty}\left[\int_{u}^{\infty} \frac{\bar{F}(x+t)}{\bar{F}(u+t)} \mathrm{d} x\right] \frac{\bar{F}(u+t)}{\bar{F}(t) \bar{H}(u)} f_{k: n}(t) \mathrm{d} t \\
& =\int_{0}^{\infty} \frac{m_{F}(u+t) \bar{F}(u+t)}{\bar{F}(t) \bar{H}(u)} f_{k: n}(t) \mathrm{d} t .
\end{aligned}
$$

Now, if $F$ is IMRL then, for all $t \geq 0, m_{F}(u+t) \geq m_{F}(u)$, and so

$$
\begin{aligned}
m_{H}(u) & \geq \int_{0}^{\infty} m_{F}(u) \frac{\bar{F}(u+t)}{\bar{F}(t) \bar{H}(u)} f_{k: n}(t) \mathrm{d} t \\
& =m_{F}(u)\left(\int_{0}^{\infty} \frac{\bar{F}(u+t)}{\bar{F}(t)} f_{k: n}(t) \mathrm{d} t\right) / \bar{H}(u) \\
& =m_{F}(u),
\end{aligned}
$$

which reveals that $X_{1} \leq{ }_{\operatorname{mrl}} X_{1}^{(k)}$. When $F$ is DMRL, the above inequality gets reversed and the required result follows immediately.

(ii) Let us assume that $F$ is DHR. Then $\bar{F}(x+t) / \bar{F}(x)$ is increasing with respect to $x$ for all $t \geq 0$. So, for every $y \geq z \geq 0$ and all $t \geq 0$, we have

$$
\frac{\bar{F}(y+t)}{\bar{F}(y)} \geq \frac{\bar{F}(z+t)}{\bar{F}(z)}
$$

and, consequently,

$$
\int_{0}^{\infty} \frac{\bar{F}(y+t)}{\bar{F}(y) \bar{F}(t)} f_{k: n}(t) \mathrm{d} t \geq \int_{0}^{\infty} \frac{\bar{F}(z+t)}{\bar{F}(z) \bar{F}(t)} f_{k: n}(t) \mathrm{d} t,
$$

or, equivalently,

$$
\frac{\bar{H}(y)}{\bar{F}(y)} \geq \frac{\bar{H}(z)}{\bar{F}(z)} .
$$

This means that $X_{1} \leq \mathrm{hr} X_{1}^{(k)}$. For the IHR case, the proof is similar and therefore omitted for the sake of brevity.

(iii) Assume that $F$ is DHR. Then, by using the results in part (ii) and Bagai and Kochar (1986) (also see Theorem 3.B.20 of Shaked and Shanthikumar (2007)), the result follows.

(iv) Suppose that $f$ is log-convex. Then $f(x+t) / f(x)$ is increasing with respect to $x$ for all $t \geq 0$. So, for $y \geq z \geq 0$ and all $t \geq 0$, we have

$$
\frac{f(y+t)}{f(y)} \geq \frac{f(z+t)}{f(z)}
$$

and, consequently,

$$
\int_{0}^{\infty} \frac{f(y+t)}{f(y) \bar{F}(t)} f_{k: n}(t) \mathrm{d} t \geq \int_{0}^{\infty} \frac{f(z+t)}{f(z) \bar{F}(t)} f_{k: n}(t) \mathrm{d} t,
$$


or, equivalently,

$$
\frac{h(y)}{f(y)} \geq \frac{h(z)}{f(z)} .
$$

This means that $X_{1} \leq_{\operatorname{lr}} X_{1}^{(k)}$. A similar proof may be presented for the case of the log-concavity of $f$.

\section{Characterizations}

Suppose that the distribution function $F$ of the original lifetimes is exponential. From (2), it is easy to see that

$$
\left(X_{1}, \ldots, X_{n-k}\right) \stackrel{\text { st }}{=}\left(X_{1}^{(k)}, \ldots, X_{n-k}^{(k)}\right),
$$

and that $X_{1}^{(k)}, \ldots, X_{n-k}^{(k)}$ are independent. Bairamov and Arnold (2008) showed that the converse of (6) is also true, that is, if two vectors $\left(X_{1}, \ldots, X_{n-k}\right)$ and $\left(X_{1}^{(k)}, \ldots, X_{n-k}^{(k)}\right)$ have the same distribution, then the distribution function $F$ of the original lifetimes is an exponential distribution (see Theorem 1 of Bairamov and Arnold (2008)). They also established the following theorem characterizing the exponential distribution based on the independence of $X_{1}^{(k)}$ and $X_{2}^{(k)}$, and some well-known ageing properties of the distribution $F$.

Theorem 2. If $X_{1}^{(k)}$ and $X_{2}^{(k)}$ are independent, and

(i) $\bar{F}(x)$ is strictly decreasing on $(0, \infty)$, and

(ii) $F$ is either IHR or DHR,

then $F$ is an exponential distribution.

In what follows, we characterize the exponential distribution of the original lifetimes based on the condition that $X_{1}^{(k)}$ and $X_{2}^{(k)}$ are uncorrelated and in this case the two conditions in Theorem 2 can be dropped for this characterization. This result would thus form a natural extension of the result in Theorem 2. To this end, we first establish the following theorem which provides a relationship between the covariance of $X_{1}^{(k)}$ and $X_{2}^{(k)}$ and the mean residual life function of the component lifetime distribution $F\left(m_{F}\right)$.

Theorem 3. For $k=1, \ldots, n-1$, we have $\operatorname{cov}\left(X_{1}^{(k)}, X_{2}^{(k)}\right)=\operatorname{var}\left(m_{F}\left(X_{k: n}\right)\right)$.

Proof. First we derive the product moment $\mathbb{E}\left(X_{1}^{(k)} X_{2}^{(k)}\right)$ as follows:

$$
\begin{aligned}
\mathbb{E}\left(X_{1}^{(k)} X_{2}^{(k)}\right) & =\int_{0}^{\infty} \int_{0}^{\infty} \mathbb{P}\left(X_{1}^{(k)}>x, X_{2}^{(k)}>y\right) \mathrm{d} x \mathrm{~d} y \\
& =\int_{0}^{\infty} \int_{0}^{\infty} \int_{0}^{\infty} \frac{\bar{F}(x+t)}{\bar{F}(t)} \frac{\bar{F}(y+t)}{\bar{F}(t)} f_{k: n}(t) \mathrm{d} t \mathrm{~d} x \mathrm{~d} y \\
& =\int_{0}^{\infty}\left(\int_{0}^{\infty} \int_{0}^{\infty} \frac{\bar{F}(x+t)}{\bar{F}(t)} \frac{\bar{F}(y+t)}{\bar{F}(t)} \mathrm{d} x \mathrm{~d} y\right) f_{k: n}(t) \mathrm{d} t \\
& =\int_{0}^{\infty}\left(\int_{0}^{\infty} \frac{\bar{F}(x+t)}{\bar{F}(t)} \mathrm{d} x\right)^{2} f_{k: n}(t) \mathrm{d} t \\
& =\int_{0}^{\infty} m_{F}^{2}(t) f_{k: n}(t) \mathrm{d} t \\
& =\mathbb{E}\left(m_{F}^{2}\left(X_{k: n}\right)\right) .
\end{aligned}
$$


From Theorem 4 of Bairamov and Arnold (2008), we also know that $\mathbb{E}\left(X_{1}^{(k)}\right)=\mathbb{E}\left(m_{F}\left(X_{k: n}\right)\right)$, and, therefore, we obtain, upon noting that $X_{1}^{(k)} \stackrel{\text { st }}{=} X_{2}^{(k)}$ for the second equality,

$$
\begin{aligned}
\operatorname{cov}\left(X_{1}^{(k)}, X_{2}^{(k)}\right) & =\mathbb{E}\left(X_{1}^{(k)} X_{2}^{(k)}\right)-\mathbb{E}\left(X_{1}^{(k)}\right) \mathbb{E}\left(X_{2}^{(k)}\right) \\
& =\mathbb{E}\left(m_{F}^{2}\left(X_{k: n}\right)\right)-\mathbb{E}^{2}\left(m_{F}\left(X_{k: n}\right)\right) \\
& =\operatorname{var}\left(m_{F}\left(X_{k: n}\right)\right),
\end{aligned}
$$

as required.

Theorem 4. If $X_{1}^{(k)}$ and $X_{2}^{(k)}$ are uncorrelated, then $F$ is an exponential distribution.

Proof. Suppose that $\operatorname{cov}\left(X_{1}^{(k)}, X_{2}^{(k)}\right)=0$. Then, by Theorem 3, we have $\operatorname{var}\left(m_{F}\left(X_{k: n}\right)\right)=$ 0 , which means that, for every $t \geq 0, m_{F}(t)=c$, where $c$ is a constant. Setting $t=0$ we get $c=\mathbb{E}\left(X_{1}\right)$, and, for every $t \geq 0$, we therefore have (see Shaked and Shanthikumar (2007, Theorem 2.A.3))

$$
\bar{F}(t)=\frac{\mathbb{E}\left(X_{1}\right)}{m_{F}(t)} \exp \left\{-\int_{0}^{t} \frac{1}{m_{F}(x)} \mathrm{d} x\right\}=\exp \left\{-\frac{t}{c}\right\},
$$

as required.

\section{Ageing properties of residual life lengths of live components}

In this section we study some ageing properties of the residual life lengths of components that are still alive after the $k$ th failure. For the ensuing discussion, it is useful to briefly give the definition and some properties of mixture distributions. Suppose that a random vector $\left(T_{1}, \ldots, T_{n}\right)$ has joint distribution function

$$
F\left(t_{1}, \ldots, t_{n}\right)=\int_{\chi} \prod_{i=1}^{n} F_{i}\left(t_{i} \mid \theta_{1}, \ldots, \theta_{r}\right) \mathrm{d} \Pi\left(\theta_{1}, \ldots, \theta_{r}\right),
$$

where $\Pi$ is an $r$-dimensional probability distribution concentrated on $\chi \subseteq \mathbb{R}^{r}$ and, for any vector $\left(\theta_{1}, \ldots, \theta_{r}\right) \in \chi, F_{i}\left(. \mid \theta_{1}, \ldots, \theta_{r}\right), i=1, \ldots, n$, are one-dimensional distribution functions. This model is known as a multivariate mixture model. If $F_{i}\left(. \mid \theta_{1}, \ldots, \theta_{r}\right)$ denotes the conditional distribution of $T_{i}(\boldsymbol{\theta})=\left(T_{i} \mid \boldsymbol{\Theta}=\boldsymbol{\theta}\right)$, and $\Pi$ denotes the distribution of $\boldsymbol{\Theta}$, then the joint distribution of $\left(T_{1}(\boldsymbol{\Theta}), \ldots, T_{n}(\boldsymbol{\Theta})\right)$ is given by (7). If $F_{i}\left(. \mid \theta_{1}, \ldots, \theta_{r}\right), i=1, \ldots, n$, are absolutely continuous for each $\left(\theta_{1}, \ldots, \theta_{r}\right) \in \chi$, with corresponding density functions $f_{i}\left(. \mid \theta_{1}, \ldots, \theta_{r}\right), i=1, \ldots, n$, then the distribution of $\left(T_{1}, \ldots, T_{n}\right)$ is also absolutely continuous, and its joint density function is given by

$$
f\left(t_{1}, \ldots, t_{n}\right)=\int_{\chi} \prod_{i=1}^{n} f_{i}\left(t_{i} \mid \theta_{1}, \ldots, \theta_{r}\right) \mathrm{d} \Pi\left(\theta_{1}, \ldots, \theta_{r}\right) .
$$

It is then easy to see that the distribution function of $T_{i}$ is

$$
F_{T_{i}}(t)=\int_{\chi} F_{i}\left(t_{i} \mid \theta_{1}, \ldots, \theta_{r}\right) \mathrm{d} \Pi\left(\theta_{1}, \ldots, \theta_{r}\right),
$$

and the corresponding density function of $T_{i}$ (if it exists) is

$$
f_{T_{i}}(t)=\int_{\chi} f_{i}\left(t_{i} \mid \theta_{1}, \ldots, \theta_{r}\right) \mathrm{d} \Pi\left(\theta_{1}, \ldots, \theta_{r}\right) .
$$


Note that, when $F_{i}\left(\cdot \mid \theta_{1}, \ldots, \theta_{r}\right)=F\left(\cdot \mid \theta_{1}, \ldots, \theta_{r}\right)$ for all $i=1, \ldots, n$, the random vector $\left(T_{1}, \ldots, T_{n}\right)$ is exchangeable. Mixture models play an important role in risk theory and reliability analysis; see, e.g. Spizzichino (2001), Belzunce et al. (2009), and Misra and Misra (2012). From (2), we readily see that the joint survival function of $X_{1}^{(k)}, \ldots, X_{n-k}^{(k)}$ can be considered as a multivariate mixture model. Let $X_{t}=(X-t \mid X>t)$, that is, $X_{t}$ denotes the residual lifetime of a component at time $t$. Then, for $x \geq 0$, the survival and density functions of $X_{t}$ are $\bar{F}_{t}(x)=\bar{F}(x+t) / \bar{F}(t)$ and $f_{t}(x)=f(x+t) / \bar{F}(t)$, respectively. Hence, (3) and (4) can be respectively written as

$$
\bar{H}(t)=\int_{0}^{\infty} \bar{F}_{t}(x) f_{k: n}(t) \mathrm{d} t
$$

and

$$
h(t)=\int_{0}^{\infty} f_{t}(x) f_{k: n}(t) \mathrm{d} t .
$$

Some ageing properties of the original lifetime distribution are known to be preserved by the residual lifetime distribution. For example, if the distribution function $F$ has a monotone hazard rate then $F_{t}$ has a monotone hazard rate for all $t \geq 0$. Also, $F_{t}$ preserves the DMRL (IMRL) and $\log$-convex (log-concave) properties of the original lifetime distribution $F$ for all $t \geq 0$. The following theorem establishes that some ageing properties of the original lifetime distribution are preserved by the residual life lengths of the live components after the $k$ th failure.

Theorem 5. We have the following ageing properties.

(i) If $F$ is IMRL, then $H$ is IMRL.

(ii) If $F$ is DHR, then $H$ is DHR.

(iii) If $f$ is log-convex, then $h$ is log-convex.

Here $H$ and $h$ are the distribution and density functions of $X_{1}^{(k)}$ presented in (8) and (9).

Proof. (i) If $F$ is IMRL then $F_{t}$ is also IMRL. But, the class of distributions that are IMRL is closed under mixtures (see Proposition D.5 of Marshall and Olkin (2007)), and the required result then follows.

(ii) If $F$ is DHR then $F_{t}$ is also DHR. From the fact that the class of distributions that are DHR is closed under mixtures (see Corollary D.4.a of Marshall and Olkin (2007)), the required result then follows.

(iii) If $f$ is log-convex then $f_{t}$ is also log-convex. So, from the closure property of logconvex density under mixtures (see Section G of Chapter 5 of Marshall and Olkin (2007) and An (1998)), the required result follows.

\section{Multivariate stochastic ordering of residual life lengths of live components in the two-sample situation}

Suppose that we have two systems, say I and II. System I is an $(n-k+1)$-out-of- $n$ system comprising $n$ components with i.i.d. lifetimes $X_{1}, \ldots, X_{n}$ from an absolutely continuous distribution $F$. Similarly, system II is an $\left(m-k^{\prime}+1\right)$-out-of- $m$ system with the $m$ lifetimes $Y_{1}, \ldots, Y_{m}$ of the components of the system being i.i.d. with common absolutely continuous distribution function $G$. Furthermore, let $X_{1}^{(k)}, \ldots, X_{n-k}^{(k)}$ and $Y_{1}^{\left(k^{\prime}\right)}, \ldots, Y_{m-k^{\prime}}^{\left(k^{\prime}\right)}$ be the residual life lengths of the live components after the $k$ th failure in system I and the $k^{\prime}$ th failure in system II, with joint distribution functions $H^{(k)}$ and $H^{\prime\left(k^{\prime}\right)}$, respectively. 
In this section we establish the multivariate stochastic ordering between the vectors of residual life lengths of live components of these two systems under some conditions. For this purpose, we will use the following theorem.

Theorem 6. (Khaledi and Kochar (2000a).) Let $X_{1}, \ldots, X_{n}$ be nonnegative independent random variables with common absolutely continuous distribution $F$, and let $Y_{1}, \ldots, Y_{m}$ be another set of nonnegative independent random variables with common absolutely continuous distribution $G$. If $X_{1} \leq \mathrm{hr} Y_{1}$ and either $F$ or $G$ is DHR, then, for $k \leq k^{\prime}$ and $n-k \geq m-k^{\prime}$, we have $X_{k: n} \leq_{\mathrm{st}} Y_{k^{\prime}: m}$.

Now, we present the main theorem of this section.

Theorem 7. Suppose that $X_{1}^{(k)}, \ldots, X_{n-k}^{(k)}$ and $Y_{1}^{\left(k^{\prime}\right)}, \ldots, Y_{m-k^{\prime}}^{\left(k^{\prime}\right)}$ are the residual life lengths of the live components in systems I and II, respectively. If $X_{1} \leq_{\mathrm{hr}} Y_{1}$ and either $F$ or $G$ is DHR, then, for $k \leq k^{\prime}$ and $n-k=m-k^{\prime}$,

$$
\left(X_{1}^{(k)}, \ldots, X_{n-k}^{(k)}\right) \leq_{\mathrm{st}}\left(Y_{1}^{\left(k^{\prime}\right)}, \ldots, Y_{m-k^{\prime}}^{\left(k^{\prime}\right)}\right) .
$$

Proof. In order to prove the desired result, we use Theorem 3.1 of Belzunce et al. (2009) which presents some sufficient conditions for the comparison of multivariate mixture models in the sense of multivariate stochastic ordering. It is easy to see that the DHR property of $F$ or $G$ is equivalent to Condition (i) in Theorem 3.1 of Belzunce et al. (2009) and that the condition $X_{1} \leq \mathrm{hr} Y_{1}$ is equivalent to Condition (ii) of the mentioned theorem. Moreover, according to Theorem 6 and in the light of the DHR property of $F$ or $G$, we find that the assumption $X_{1} \leq_{\mathrm{hr}} Y_{1}$ implies that $X_{k: n} \leq_{\mathrm{st}} Y_{k^{\prime}: m}$ for $k \leq k^{\prime}$ and $n-k=m-k^{\prime}$. With this observation and (2), Condition (iii) in Theorem 3.1 of Belzunce et al. (2009) is obtained, thus completing the proof.

For a given positive integer $i=1, \ldots, n-k+1, k \leq n$, let

$$
V_{k: n}^{(i)}=X_{i+k-1: n}-X_{k-1: n} \quad \text { and } \quad W_{k: n}^{(i)}=Y_{i+k-1: n}-Y_{k-1: n}
$$

respectively denote the $i$-spacings of samples $X_{1}, \ldots, X_{n}$ and $Y_{1}, \ldots, Y_{n}$, with $X_{0: n} \equiv$ $Y_{0: n} \equiv 0$. In particular, for $i=1$, we have

$$
\tilde{V}_{k: n}^{(1)}=(n-k+1)\left(X_{k: n}-X_{k-1: n}\right) \quad \text { and } \quad \tilde{W}_{k: n}^{(1)}=(n-k+1)\left(Y_{k: n}-Y_{k-1: n}\right)
$$

as the corresponding normalized 1-spacings. In reliability theory, $X_{n-k+1: n}$ represents the lifetime of the $k$-out-of- $n$ system, and $V_{k: n}^{(i)}$ then gives the additional lifetime to be gained if the $(n-k-i+2)$-out-of- $n$ system is used rather than a $(n-k+2)$-out-of- $n$ system. The reader is referred to Kirmani (1996) and Hu and Zhuang (2006) for some additional details. In stochastic auction theory, the mean of the sample spacing $V_{k: n}^{(1)}$ gives the expected rent of the winner in either a buyer's $k$-auction or a reverse $k$-auction. For further details on auction theory, see Paul and Gutierrez (2004) and Li (2005). By using the definition of the residual life lengths and (5), we can interpret the $i$-spacing as follows. Suppose that we have an $(n-k+2)$-out-of- $n$ system. After the $(k-1)$ th failure, $n-k+1$ components are still alive. If we reuse these remaining components in another $(n-k-i+2)$-out-of- $(n-k+1)$ system then the $i$-spacing represents the lifetime of this latter system. Recently, several authors have discussed stochastic comparisons of $i$-spacings. Bartoszewicz (1986) proved that if $X_{1} \leq_{\text {disp }} Y_{1}$ then $V_{k: n}^{(1)} \leq_{\text {st }} W_{k: n}^{(1)}$. Kochar (1999) showed that if $X_{1} \leq \mathrm{lr} Y_{1}$ and either $F$ or $G$ is DHR, then $V_{k: n}^{(1)} \leq \mathrm{hr} W_{k: n}^{(1)}$. Subsequently, Khaledi 
and Kochar (1999) proved that if $X_{1} \leq \mathrm{hr} Y_{1}$ and either $F$ or $G$ is DHR, then, for $k \leq k^{\prime}$ and $n-k \geq m-k^{\prime}$, we have $\tilde{V}_{k: n}^{(1)} \leq$ st $\tilde{W}_{k^{\prime}: m}^{(1)}$. They also showed that if $X_{1} \leq \operatorname{lr} Y_{1}$ and either $F$ or $G$ have $\log$-convex density, then $\tilde{V}_{k: n}^{(1)} \leq_{\operatorname{lr}} \tilde{W}_{k^{\prime}: m}^{(1)}$ for $k \leq k^{\prime}$ and $n-k=m-k^{\prime}$. Xu and Li (2006) generalized this result and proved that if $F$ or $G$ have log-convex density, then $V_{k: n}^{(l)} \leq \operatorname{lr} W_{k^{\prime}: m}^{(l)}$ for $k \leq k^{\prime}$ and $n-k \geq m-k^{\prime}$. Hu and Wei (2001) established a more general result of the following form: if $X_{1} \leq \mathrm{hr} Y_{1}$ and either $F$ or $G$ is DHR, then $V_{k: n}^{(q-k+1)} \leq_{\mathrm{st}} W_{k^{\prime} \cdot m}^{\left(l-k^{\prime}+1\right)}$ for $l \geq k-k^{\prime}$ and $l-q \geq k^{\prime}-k \geq n-m$. Using a discussion similar to that presented in Theorem 6.B.2 of Shaked and Shanthikumar (2007), the following corollary can be obtained from Theorem 7.

Corollary 1. Let $X_{1}, \ldots, X_{n}$ be a random sample of size $n$ from an absolutely continuous distribution $F$, and let $Y_{1}, \ldots, Y_{m}$ be a random sample of size $m$ from another absolutely continuous distribution $G$. If $X_{1} \leq \mathrm{hr} Y_{1}$ and either $F$ or $G$ is DHR, then, for $k \leq k^{\prime}, n-k=$ $m-k^{\prime}$, and $1 \leq i \leq j \leq n-k$, we have $V_{k+1: n}^{(i)} \leq_{\mathrm{st}} W_{k^{\prime}+1: m}^{(j)}$.

Note that Corollary 1 is a special case of the result of $\mathrm{Hu}$ and Wei (2001).

\section{Acknowledgements}

The authors express their sincere thanks to the editor and anonymous reviewer for their useful comments and suggestions on an earlier version of the paper which led to this improved version. The second and third authors would like to express the deepest appreciation to Professor Baha-Eldin Khaledi who introduced them to the interesting area of stochastic orderings.

\section{References}

AN, M. Y. (1998). Logconcavity versus logconvexity: a complete characterization. J. Econom. Theory 80, 350-369.

Arnold, B. C., Balakrishnan, N. and Nagaraja, H. N. (1992). A First Course in Order Statistics. John Wiley, New York.

Bagai, I. AND Kochar, S. C. (1986). On tail-ordering and comparison of failure rates. Commun. Statist. Theory Meth. 15, 1377-1388.

Bairamov, I. And Arnold, B. C. (2008). On the residual lifelengths of the remaining components in an $n-k+1$ out of $n$ system. Statist. Prob. Lett. 78, 945-952.

Barlow, R. E. AND Proschan, F. (1981). Statistical Theory of Reliability and Life Testing. Holt, Rinehart and Winston, New York.

Bartoszewicz, J. (1986). Dispersive ordering and the total time on test transformation. Statist. Prob. Lett. 4, $285-288$.

Belzunce, F., Mercader, J.-A., Ruiz, J.-M. and Spizzichino, F. (2009). Stochastic comparisons of multivariate mixture models. J. Multivariate Anal. 100, 1657-1669.

Boland, P. J., El-Neweihi, E. And Proschan, F. (1994). Applications of the hazard rate ordering in reliability and order statistics. J. Appl. Prob. 31, 180-192.

Boland, P. J., Shaked, M. and Shanthikumar, J. G. (1998). Stochastic ordering of order statistics. In Order Statistics: Theory \& Methods (Handbook Statist. 16), North-Holland, Amsterdam, pp. 89-103.

CRAmER, E. AND KAMPs, U. (1996). Sequential order statistics and $k$-out-of- $n$ systems with sequentially adjusted failure rates. Ann. Inst. Statist. Math. 48, 535-549.

David, H. A. And Nagaraja, H. N. (2003). Order Statistics, 3rd edn. John Wiley, Hoboken, NJ.

Hu, T. AND WeI, Y. (2001). Stochastic comparisons of spacings from restricted families of distributions. Statist. Prob. Lett. 53, 91-99.

Hu, T. and Zhuang, W. (2006). Stochastic comparisons of $m$-spacings. J. Statist. Planning Infer. 136, $33-42$.

Kamps, U. (1995). A Concept of Generalized Order Statistics. Teubner, Stuttgart.

Khaledi, B.-E. AND Kochar, S. (1999). Stochastic orderings between distributions and their sample spacings. II. Statist. Prob. Lett. 44, 161-166.

KHaLedi, B.-E. AND KochaR, S. (2000a). On dispersive ordering between order statistics in one-sample and two-sample problems. Statist. Prob. Lett. 46, 257-261.

Khaledi, B.-E. And Kochar, S. (2000b). Some new results on stochastic comparisons of parallel systems. J. Appl. Prob. 37, 1123-1128. 
KIRMANI, S. N. U. A. (1996). On sample spacings from IMRL distributions. Statist. Prob. Lett. 29, 159-166. (Correction: 29 (1997), 159-166.)

Kochar, S. C. (1999). On stochastic orderings between distributions and their sample spacings. Statist. Prob. Lett. 42, 345-352.

LAI, C.-D. AND XIE, M. (2006). Stochastic Ageing and Dependence for Reliability. Springer, New York.

LI, X. (2005). A note on expected rent in auction theory. Operat. Res. Lett. 33, 531-534.

Marshall, A. W. And OlKin, I. (2007). Life Distributions. Springer, New York.

Misra, A. K. ANd Misra, N. (2012). Stochastic properties of conditionally independent mixture models. J. Statist. Planning Infer. 142, 1599-1607.

Müller, A. And Stoyan, D. (2002). Comparison Methods for Stochastic Models and Risks. John Wiley, Chichester.

PĂLtĂneA, E. (2008). On the comparison in hazard rate ordering of fail-safe systems. J. Statist. Planning Infer. 138, 1993-1997.

Paul, A. And Gutierrez, G. (2004). Mean sample spacings, sample size and variability in auction-theoretic framework. Operat. Res. Lett. 32, 103-108.

Pledger, G. And Proschan, F. (1971). Comparisons of order statistics and of spacings from heterogeneous distributions. In Optimizing Methods in Statistics, Academic Press, New York, pp. 89-113.

Proschan, F. And Sethuraman, J. (1976). Stochastic comparisons of order statistics from heterogeneous populations, with applications in reliability. J. Multivariate Anal. 6, 608-616.

Shaked, M. And Shanthikumar, J. G. (2007). Stochastic Orders. Springer, New York.

SpIzzichino, F. (2001). Subjective Probability Models for Lifetimes. Chapman \& Hall/CRC, Boca Raton, FL.

XU, M. AND LI, X. (2006). Likelihood ratio order of m-spacings for two samples. J. Statist. Planning Infer. 136, 4250-4258.

Zhao, P. ANd Balakrishnan, N. (2009). Characterization of MRL order of fail-safe systems with heterogeneous exponential components. J. Statist. Planning Infer. 139, 3027-3037.

Zhao, P., Li, X. AND Balakrishnan, N. (2009). Likelihood ratio order of the second order statistic from independent heterogeneous exponential random variables. J. Multivariate Anal. 100, 952-962. 